\title{
Psychrotolerant methanogenic archaea: Diversity and cold adaptation mechanisms
}

\author{
DONG XiuZhu* \& CHEN ZiJuan \\ State Key Laboratory of Microbial Resources, Institute of Microbiology, Chinese Academy of Sciences, Beijing 100101, China
}

Received February 28, 2012; accepted April 10, 2012

\begin{abstract}
Because of their diversity and abundance in a wide range of environments, particularly in cold regions, cold-adaptive archaea are expected to play a pivotal role in material recycling in cold environments. Methanogenic archaea are ubiquitous on earth and produce a large amount of methane $\left(\mathrm{CH}_{4}\right)$ as their main carbon metabolite. Methanogens are the most laboratory amendable archaea. The few psychrophilic archaea that have been cultured to date are mainly affiliated with methanogens, thus make them a good model for investigating mechanisms of archaeal cold adaptation. Studies of psychrotolerant methanogens have been ongoing since the 1990s. Using Methanococcoides burtonii, a methanogen isolated from Ace Lake in Antarctica, extensive studies on the genomic characteristics associated with cold adaptation have been carried out by the Cavicchioli laboratory. We recently analyzed the genome of another psychrophilic methanogen and identified the gene repertoire associated with cold adaptation. This review summarizes recent studies of psychroactive methanogens, particularly their diversity, the genomics and proteomics associated with their cold adaptation, and the cellular components and proteins likely involved in their cold protection.
\end{abstract}

psychrotolerant methanogens, diversity, cold adaptation mechanisms

Citation: Dong X Z, Chen Z J. Psychrotolerant methanogenic archaea: Diversity and cold adaptation mechanisms. Sci China Life Sci, 2012, 55: 415-421, doi: $10.1007 / \mathrm{s} 11427-012-4320-0$

\section{Methanogenic archaea}

Methanogenic archaea (methanogens) are currently the only organisms known to produce abundant methane $\left(\mathrm{CH}_{4}\right)$ as an end metabolite and to use methanogenesis as an exclusive energy conservation approach [1]. Methanogenic archaea have long been studied not only because they are the most commonly cultured and amenable representatives of archaea, but also owing to the ecological impact of their function on the global methane cycle. Indeed, it is estimated that microbial processes mediate $85 \%$ of annual global methane formation and $60 \%$ of $\mathrm{CH}_{4}$ consumption [2]. However, only the activities of three key groups of microorganisms have

*Corresponding author (email: dongxz@im.ac.cn) been shown to contribute to the cycle: (i) $\mathrm{CH}_{4}$ production by methanogenic archaea; (ii) $\mathrm{CH}_{4}$ oxidation by aerobic methylotrophic bacteria; and (iii) anaerobic methane oxidation (AOM), which occurs within the continental boundaries of the oceans and is driven by specific methanogenic archaeal groups.

Terrestrial methane formation is believed to primarily occur in natural wetlands, especially in cold regions. Approximately $75 \%$ of the earth's biosphere is subject to permanently low temperatures [3], and permafrost has been estimated to contribute about $25 \%$ of the methane released from natural sources. However, the methanogens that inhabit in cold wetlands and their cold adaptation mechanisms are poorly understood. This review covers the rapid progress toward characterization of the diversity of cold-adaptive methanogenic archaea and highlights the current under- 
standing of their cold adaptation biology via studies of their genomics, functional proteins and cellular components.

\section{Psychrophilic vs. psychrotolerant}

Cold-adaptive organisms are known as stenopsychrophiles or eurypsychrophiles. The former refers to microorganisms that grow exclusively at low temperatures (usually below $20^{\circ} \mathrm{C}$ ) and cannot tolerate higher temperatures, while the latter term, which is synonymous with psychrotolerant organisms, describes mesophilic microorganisms that can tolerate low temperatures [3]. However, the precise classification of psychrophilic and psychrotolerant organisms has been continuously debated since the 1960s. Currently, both maximal growth temperature $\left(T_{\max }\right)$ and optimal growth temperature $\left(T_{\mathrm{opt}}\right)$ are considered when determining the temperature characteristics of a microorganism. Based on this classification, psychrophiles exhibit a $T_{\text {opt }}$ below $20^{\circ} \mathrm{C}$ and a $T_{\max }$ of $25^{\circ} \mathrm{C}$; while psychrotolerant organisms include those that have a $T_{\text {opt }}$ above $30^{\circ} \mathrm{C}$, but can survive at $0-5^{\circ} \mathrm{C}$.

\section{Diversity of psychrotolerant methanogens}

Methanogens are highly sensitive to oxygen and therefore only distributed in anoxic environments. To date, most of the described methanogens that have been described to date have optimal growth temperatures greater than $37^{\circ} \mathrm{C}$ and as high as $100^{\circ} \mathrm{C}$, while very few are psychrotolerant (Table 1). The psychrotolerant methanogens have mostly been isolated from aquatic niches in boreal or polar regions and show the highest growth rates at $18-35^{\circ} \mathrm{C}$ [4-12]. Phylogenetically, these organisms are restricted to 2 out of the 5 methanogenic phyla, Methanosarcinaceae (Methanococcoides and Methanosarcina) and Methanomicrobiales (Methanogenium). However, the release of large amounts of $\mathrm{CH}_{4}$ from the bo- real regions of earth is indicative of abundant methanogens active in cold environments. We recently isolated a novel psychrophilic methanogen, Methanolobus psychrophilus $\mathrm{R} 15$, from a cold freshwater wetland situated in the Tibetan plateau. The strain is methylotrophic and phylogenetically affiliated with Methanosarcinaceae [10]. This organism grows at temperatures ranging from 0 to $25^{\circ} \mathrm{C}$, but optimally at $18^{\circ} \mathrm{C}$.

Cold-adaptive methanogens are ubiquitous in wetlands, freshwater sediments, landfills, farms, permafrost, and the basal ice layer of glaciers [3]. Although only a few psychrophilic or psychrotolerant methanogenic species have been cultured and described to date, their diverse and prevalent populations in cold areas have been estimated by molecular ecological approaches. Using molecular ecological approaches, Kotsyurbenko et al. [13] showed that Methanosarciaceae, Methanomicrobiaceae, and Rice Cluster II were dominant in an acidic Siberian peat bog. Studies of different types of boreal wetlands with acidic $\mathrm{pH}$ in Finland revealed distinct methanogenesis pathways and methanogen communities [14]. Specifically, $\mathrm{H}_{2}$ and $\mathrm{CO}_{2}$ contributed higher proportions of the methane production in oligotrophic fens, but lower in mesotrophic fens. In their study, methanogens affiliated with Methanosaeta spp. were dominant in mesotrophic fens, while those of rice cluster-I were prevalent in oligotrophic fens, suggesting that methanogenesis pathways and distinct methanogen communities are most likely correlated to the vegetation present in each region. By constructing both $16 \mathrm{~S}$ rRNA and $m c r A$ gene libraries, we determined that methanogens affiliated with 4 out of the 5 phyla are dominant in the cold Zoige wetland soil of the Tibetan Plateau, and only a few other archaeal phyla coexist in this region (Figure 1) [15].

By determining the methanogenesis rates from various methanogenic substrates using the Zoige wetland soil as inoculants at low temperature $\left(15^{\circ} \mathrm{C}\right)$, as well as measuring the accumulated methanogenic precursors in the methanogenesis-inhibited Zoige wetland soil samples, we found that

Table 1 Methanogens isolated from cold environments

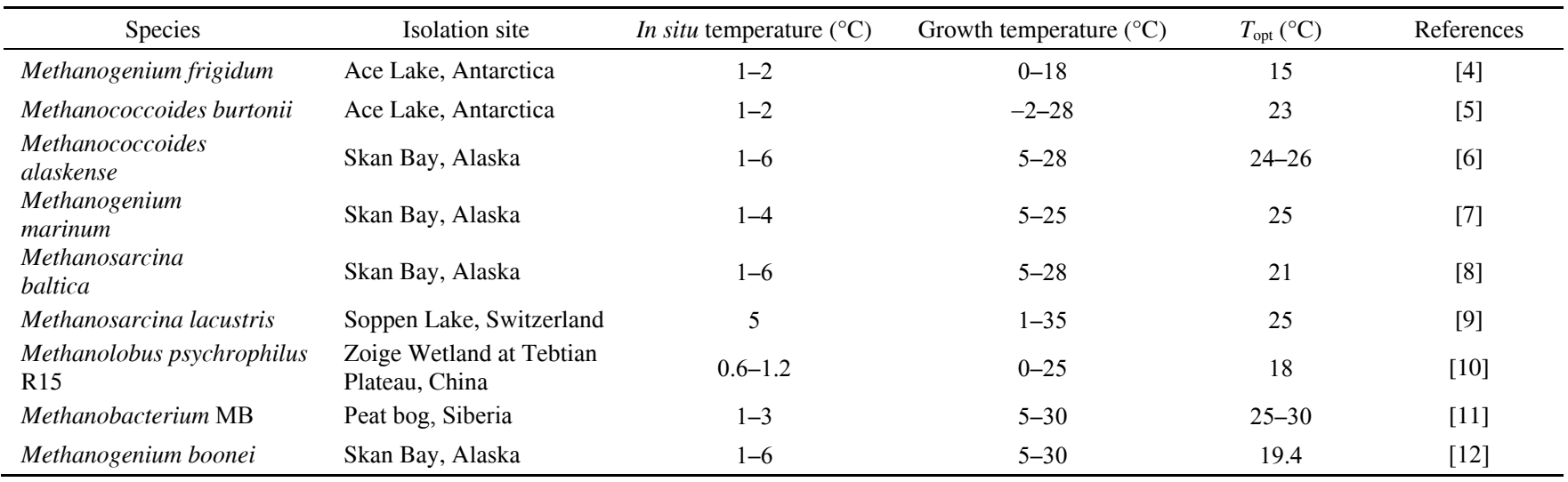




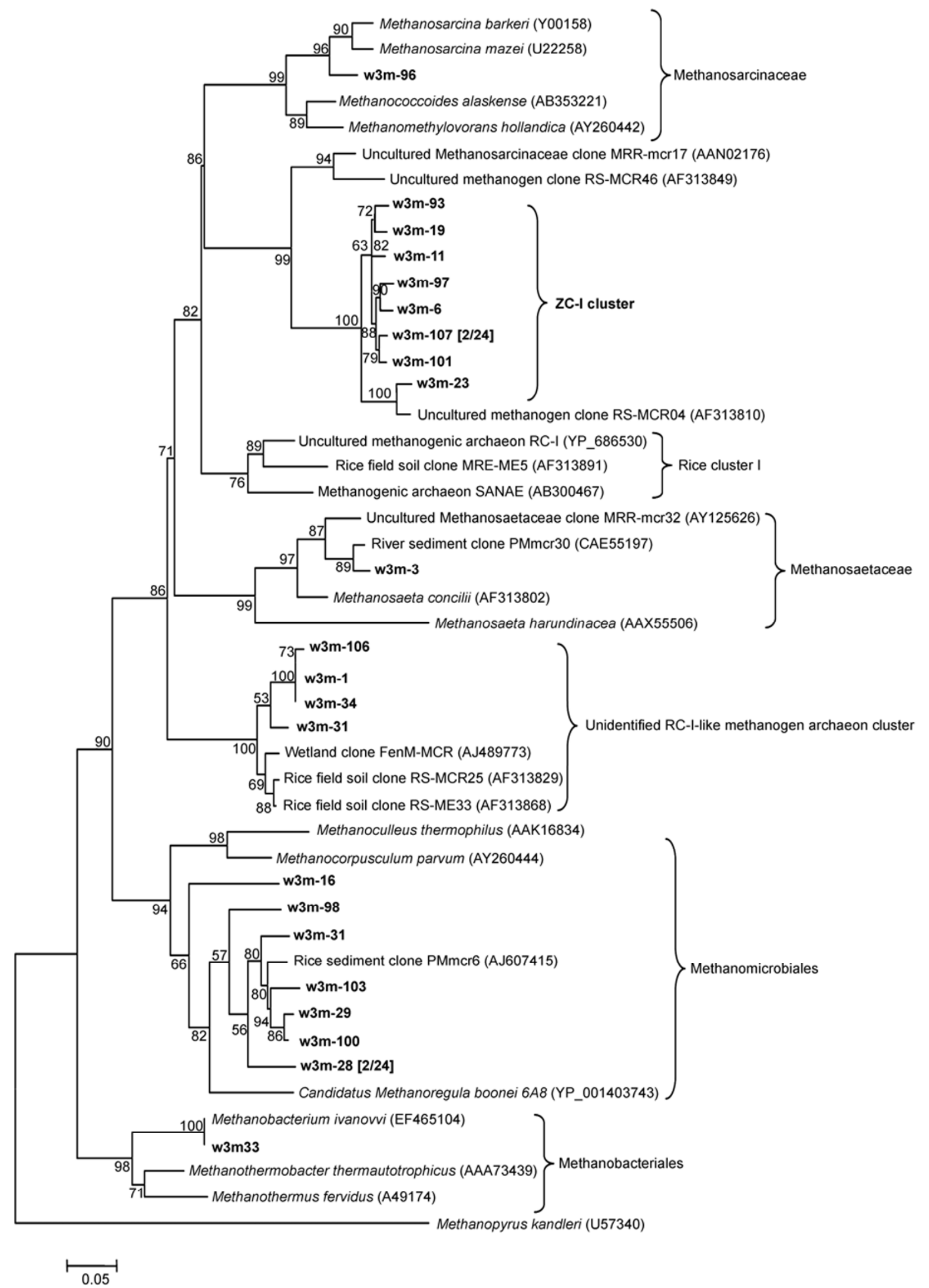

Figure 1 Phylogenetic tree of methanogen $m c r A$ sequences retrieved from Zoige wetland soil based on the homology of deduced sequences of 158 amino acids (from [15]). Those in bold face are identified from Zoige wetland soil. 
acetate and methanol are important methanogenic precursors in the Zoige wetland [16]. Combining the survey of methanogen diversity in the same wetland, we believe that though the cold-adaptive methanogens were scattered throughout most of the phyla, the predominant species of psychrophilic methanogens are more related to methylotrophs in the Zoige wetland.

\section{Cold adaptation mechanisms}

Psychrophilic organisms generally have to be equipped with the overall cold-adaptive cellular components to enable proper performance of cellular processes that ensures their survival in cold environments. Therefore, cold-adaptive mechanisms involve cellular components and functional machinery or proteins. These include cell wall components, outer membranes (lipid composition), cellular machines (ribosomes), enzymes, nucleic acids (tRNA), and chaperones such as cold shock proteins/domains (CSP/CSD) and TRAM domain proteins.

Studies of the cold-adaptive mechanism of methanogenic archaea began shortly after they were discovered in the 1990s and were mainly conducted by Cavicchioli laboratory. They used the psychrotolerant methanogen Methanococcoides burtonii, a strain isolated from an Antarctica Lake, as their main subject for comprehensive studies of cold-adaptive mechanisms conducted using genomic and biochemical approaches. We recently analyzed the cold-adaptive gene profile for a psychrophilic methanogen, Methanolobus psychrophilus R15, based on comparative genomics and transcriptomics studies. Although the overall cold-related gene repertoires of the two methanogens were similar, there were some different cold-adaptive protein categories. Specifically, there were more protein families for oxygen detoxification, two-component-systems for signal transduction, and thermosomes likely to play a role in cold adaptation.

\subsection{Cold adaptive cellular components}

\subsubsection{Membrane lipids}

It is well known that cold temperatures may be lethal to microorganisms, causing their cellular membrane lipids to become rigid and inactivating important ion channels and integrated enzymes. While resembling cold-adaptive bacteria, the cold-adaptive $M$. burtonii avoids these effects by increasing its levels of membrane-lipid unsaturation to maintain the fluidity of its membrane at lower temperatures [17]. Proteomics-based enzyme identification of $M$. burtonii has also revealed that this organism contains homologs of the plant geranylgeranyl reductase involved in lipid biosynthesis instead of a bacteria-like desaturase mechanism [17]. This further supports the phylogenetic relationship between archaea and eukaryotes.

\subsubsection{Compatible solutes}

Compatible solutes in cellular cytoplasm refer to small organic osmolytes that include, but are not limited to, sugars, polyols, amino acids, and their derivatives [18]. Compatible solutes are compatible with cell metabolism, even at molar concentrations, and exist widely in the three domains of organisms, but show species-based preferred compound categories. Glycine betaine, some amino acids and their derivatives are the likely compatible solutes of methanogens [18]. However, we found that a type of adenosine derivate is increased in the $4^{\circ} \mathrm{C}$-cultured and higher saline-cultured cell extracts of $M$. psychrophilus R15, implying that a new type of compatible solute may play a role in cold adaptation.

Initial studies of compatible solutes focused on their roles in bacteria or yeast in adaptation to high salinity stress. However, recent studies have suggested that they play significant roles in biomolecule protection in vitro [19,20], such as stabilization of protein and nucleic acid structures at low temperatures [19]. Therefore, it is reasonable to assume that compatible solutes not only function as osmoprotectants, but also as cryoprotectants. This hypothesis has been confirmed in E. coli [21]. Other researchers have found that cold temperatures can motivate compatible solute transportation or synthesis in Psychrobacter cryohalolentis [22]. Studies have also shown that transport proteins for the uptake of glycine betaine by $M$. burtonii are much more highly expressed at $4^{\circ} \mathrm{C}$ than at $23^{\circ} \mathrm{C}$ [23]. M. burtonii has previously been shown to modify compatible solute compositions at different growth temperatures [24]. Therefore, compatible solutes are also likely important in resisting cold stress.

\subsection{Cold adaptive genomic characteristics}

The genome outline of archaea relevant to cold-adaptive mechanisms is mostly gained from the Antarctic methanogens Methanococcoides burtonii and Methanogenium frigidum. Saunders et al. compared the genomic features of these two cold-adaptive methanogens to those of methanogens with $T_{\text {opt }}$ values of $37-98^{\circ} \mathrm{C}$. Their analysis revealed unique genes for $M$. burtonii and M. frigidum. Among these genes, the one with the highest reliability was predicted to be a 3-helical bundle DNA/RNA-binding protein [25]. In addition, proteins from the two Antarctic methanogens were found to have higher levels of non-charged polar amino acids, particularly Gln and Thr, and lower levels of hydrophobic amino acids, especially Leu. Prediction of their tRNA structure revealed that GC-content was not as important to their stability as previously believed, despite it being a key factor for hyperthermophlies [26]. Allen et al. [27] recently used the completed genome of $M$. burtonii to demonstrate that genes with psychrophilic-features could fall into the same categories as defense mechanisms and motility. Those overrepresented in the former were genes encoding type-I restriction modification systems and $\mathrm{ABC}$ 
transporters.

Goodchild et al. [28] conducted a proteomics overview of cold adaptation-associated proteins in $M$. burtonii that revealed a cold regulative pattern for the enzymes involved in both carbon and nitrogen metabolism. Specifically, they found that at least 11 proteins associated with methanogenesis and energy generation, a trimethylamine methyltransferase, and $\mathrm{F}_{420} \mathrm{H}_{2}$ dehydrogenase, were all up-regulated at lower temperatures, while two trimethylamine corrinoid proteins, dimethylamine corrinoid proteins, and a $\beta$ chain of ATP synthase were present at reduced levels under cold conditions. They also found that both methyltransferase and adenosyl homocysteinase, which removes methyltransferase inhibitor, were up-regulated at $4^{\circ} \mathrm{C}$, confirming that methyl transfer was cold active reaction. Therefore, methylotrophic methanogenesis can be predicted as a cold active pathway. Cold-regulated proteins also include those involved in coenzyme metabolism, amino acid and nucleic acid metabolism, glycolysis or gluconeogenesis, and protein modification [28-30].

Williams et al. [23] recently used differential quantitative proteomics with post-incorporation isobaric labeling (iTRAQ) and tandem liquid chromatography-mass spectrometry (LC/LC-MS/MS) to determine the overall proteomics of Methanococcoides burtonii in response to low temperatures. They found that soluble cytoplasmic, membrane-integrated, and surface associated proteins were included. Furthermore, similar to Goodchild et al. (2004), they found that numerous surface proteins were more abundant at $4{ }^{\circ} \mathrm{C}$ than at $23^{\circ} \mathrm{C}$, including secreted and membrane-anchored proteins bearing an N-terminal signal peptide and a $\mathrm{C}$-terminal membrane anchor [28]. These findings implied that extensive remodeling of the cell envelope architecture occurs in response to cold conditions. Other abundant surface proteins present at $4^{\circ} \mathrm{C}$ included those possessing domains/motifs associated with cell adhesion, such as cohesin and dockerin domains. Cells also appeared to be inclined to be closer to each other in response to cold surroundings. Indeed, larger cell aggregates of $M$. burtonii were observed at $4^{\circ} \mathrm{C}$ [28]. Similar observations were also made with $M$. psychrophilus R15. Specifically, the cell surface protein-encoding genes of this organism all increased and cells were inclined to form clumps attached to the bottom of serum bottles when cultured at $4^{\circ} \mathrm{C}$. Furthermore, Williams et al. found that three proteins, each with a predicted RNA-binding domain and a single TRAM domain identified in two distinct tRNA-modifying enzyme families, TRM2 and MiaB, were up-regulated at $4^{\circ} \mathrm{C}$ [23]. A number of oxidative stress proteins, such as thiamine biosynthesis protein (ThiC), were more abundant at $23^{\circ} \mathrm{C}$ [23]. Even more antioxidative gene categories were observed in strain R15, which not only carried the superoxide reductase (SOR)-dependent anaerobic oxides-decomposition pathway [31], but also an aerobe-characteristic reactive oxygen species (ROS)-removing mechanism composed of superoxide dismutase (SOD) and catalase. These findings suggested that elevated oxidative stress attributed to the increased metabolic activity at higher temperatures. Although reactive oxygen species could not be generated in anaerobes, non-oxygen radicals such as S-centered thiyl radicals could be generated by methylcoenzyme $\mathrm{M}(\mathrm{CoM})$ reducatase [23].

\subsection{Cold-adaptive chaperone proteins}

csp genes are present in most bacteria, but absent from most archaeal genomes [26], like that of Methanococcoides burtonii. However, two hypothetical proteins containing CSD were observed in that genome. Giaquinto et al. [32] screened a csp in the genome of Methanogenium frigidum, which is the archaeon with the lowest known upper growth temperature, and determined that archaeal Csp functioned as a cold-protective protein for a cold-sensitive growth defect in Escherichia coli. The Csp protein of Methanogenium frigidum was predicted to have higher levels of solvent-exposed basic residues than non-complementary proteins, with the basic residues located on the nucleic acid binding surface [32]. The acidic protein characterized by binding of single-stranded RNA of E. coli was small. Studies have shown that some Csp and CSD fold proteins have retained sufficient similarity throughout evolution in archaea by being able to function effectively in bacteria, and that the function of archaeal proteins are related to cold adaptation [33,34]. We also found a bacterial csp gene homologue in the recently completed genome of Methanosaeta harundinacea, a methanogen archaeon determined to be a psychrotolerant organism by producing $\mathrm{CH}_{4}$ at temperatures as low as $4^{\circ} \mathrm{C}$. However, cold-induced expression of this csp gene was not detected.

Protein translation has been determined to be the fundamental biological process sensitive to cold shock or growth under cold conditions in bacteria [21,22], while the ribosome was believed to be the cellular component involved in sensing cold. Hence, Thomas and Cavicchioli compared the structures of elongation factor 2 (EF-2) proteins, a GTPase promoting translocation of the ribosome, from psychrophilic, mesophilic, and thermophilic methanogens. They determined that amino acid residues were important determinants of protein adaptation to cold and concluded that structural features of cold-adaptive EF-2 proteins consisted of fewer salt bridges, less packed hydrophobic cores, and reduced levels of proline residues in loop structures, which enabled structural flexibility under low temperatures [35]. Furthermore, investigation of the effects of temperature on the stability and activity of EF-2 protein isolated from $M$. burtonii suggested that EF-2 was involved in cold active biochemical processes because it exhibited lower activation energy for GTP hydrolysis and protein unfolding than its thermophilic homolog [36,37]. Considering that EF-G, the bacterial counterpart of EF-2, was also determined to be a chaperone [31], it is reasonable to assume that EF-2 exists 
as a ribosome chaperone to assist its activity under cold conditions because its GTPase activity declines unless a ribosome exists.

DEAD-box RNA helicases are highly conserved enzymes that play roles in RNA metabolic processes from transcription to degradation in all three domains lives. The DEAD RNA helicase gene $($ deaD) is the first cold-related gene to be extensively studied in archaea. Based on the protein in unwinding duplex RNA in bacteria and eukaryotes, Lim et al. characterized a putative DEAD-box RNA helicase from Methanococcoides burtonii. They found that the putative deaD was highly expressed at $4^{\circ} \mathrm{C}$, but undetectable at the optimal growth temperature $\left(23^{\circ} \mathrm{C}\right)$, suggesting its cold-regulative feature [38]. Further characterization of the gene revealed an elementary composition similar to its bacterial counterparts; namely, an ATP-hydrolysis domain, RNA-binding motif, and cold-box element in the 5'-untranslated region (5'-UTR) [38].

In addition, thermosomes including GroES/EL genes were all up-regulated in the $4^{\circ} \mathrm{C}$ culture of $\mathrm{R} 15$, and were clustered with a putative DEAD-box RNA helicase gene. Thermosomes may interact with the DEAD-box RNA helicase to facilitate the decay of aberrant RNA. GroEL in $E$. coli has been determined to be a component of a mRNA protection complex [39], and Ruggero et al. [40] reported its activity as an RNA-binding protein in a crenarchaeon, Sulfolobus solfataricus. However, thermosomes appear to have played a similar role to heat shock proteins in M. burtonii, as they were all up-regulated in organisms cultured at $23^{\circ} \mathrm{C}[23]$.

To date, TRAM domain-small proteins have been restricted in archaea and conserved across euryarchaeota genomes. Anantharaman et al. [41] reported TRAM as a predicted RNA-binding domain commonly present in RNAmodifying enzymes and other proteins associated with translation machinery. Therefore, it is reasonable to assume that TRAM domain proteins function as a type of RNA chaperone analogous to bacterial Csps.

Recently, Williams et al. [42] analyzed protein profiles corresponding to incremental temperatures that spanned the growth temperature range for $M$. burtonii using 8-plex iTRAQ proteomics. Based on the functional categories of the proteins when marked differential expression was detected, they defined three distinct physiological states for the methanogen. When cells are subjected to cold stress $\left(-2^{\circ} \mathrm{C}\right)$, exosomes and RNases become active to remove the aberrant RNAs produced in the cold. Cells are also subject to oxidative stress under cold conditions; therefore, the expression of multiple antioxidative responsive proteins is elevated, including the expression of SOR, flavoproteins, and catalase. When in the cold adaptation state $\left(1-16^{\circ} \mathrm{C}\right)$, cell surface proteins those carry the cohesin and dockerin domains are highly expressed. Although growth can reach the maximum at $23-28^{\circ} \mathrm{C}, \mathrm{M}$. burtonii is under a heat shock state at these temperatures, resulting in chaperones such as
DnaK and thermosomes being abundant.

\section{Conclusion}

Although cold adaptation mechanisms among the prokaryotes can generally be considered similar based on the knowledge of the very limited psychrotolarent archaeal species so far, extensive study on more archaea may disclose a wide variety of cold adaptation strategies as they use distinct metabolic pathways, especially in chemolithotrophs. Understanding the cold adaptation mechanisms of archaea may facilitate acquisition of more archaea by generating appropriate culture conditions for psychrophilic or psychrotolerant archaea and shed light on understanding the evolution of cold adaptation mechanisms among the distinct phyla. Moreover, future biotechnological innovations would lie in archaeal metabolic functions; hence, understanding their cold adaptations may improve psychrophile-based industrial processes.

1 Boone D R, Whitman W B, Rouvière P. Diversity and taxonomy of methanogens. In: Ferry J G, ed. Methanogenesis. New York \& London: Chapman \& Hall, 1993. 35-80

2 Thauer R K, Kaster A K, Seedorf H, et al. Methanogenic archaea: ecologically relevant differences in energy conservation. Nat Rev Microbiol, 2008, 6: 579-591

3 Cavicchioli R. Cold-adapted archaea. Nat Rev Microbiol, 2006, 4: 331-343

4 Franzmann P, Liu Y, Balkwill DL, et al. Methanogenium frigidum sp. nov., a psychrophilic, $\mathrm{H}_{2}$ - using methanogen from Ace Lake, Antarctica. Int J Sys Evol Microbiol, 1997, 47: 1068-1072

5 Franzmann P D, Stringer N, Ludwig W, et al. A methanogenic archaeon from Ace Lake, Antarctica: Methanococcoides burtonii sp. nov. Sys Appl Microbiol, 1992, 15: 573-581

6 Singh N, Kendall M M, Liu Y, et al. Isolation and characterization of methylotrophic methanogens from anoxic marine sediments in Skan Bay, Alaska: description of Methanococcoides alakenese sp. nov., and emended description of Methanosarcina baltica. Int J Sys Evol Microbiol, 2005, 55: 2531-2538

7 Chong S C, Liu Y, Cummins M, et al. Methanogenium marinum sp. nov., a $\mathrm{H}_{2}$-using methanogen from Skan Bay, Alaska, and kinetics of $\mathrm{H}_{2}$ utilization. Int J Sys Evol Microbiol, 2002, 81: 263-270

8 Von Klein D, Arab H, Vlker H, et al. Methanosarcina baltica, sp. nov., a novel methanogen isolated from the Gotland Deep of the Baltic Sea. Extremophiles, 2002, 6: 103-110

9 Simankova M V, Parshina S N, Tourova T P, et al. Methanosarcina lacustris sp. nov., a new psychrotolerant methanogenic archaeon from anoxic lake sediments. Sys Appl Microbiol, 2001, 24: 362-367

10 Zhang G, Jiang N, Liu X, et al. Methanogenesis from methanol at low temperature by a novel psychrophilic methanogen, Methanolobus psychrophilus sp. nov., prevalent in Zoige wetland of Tibetan Plateau. Appl Environ Microbiol, 2008, 74: 6114-6120

11 Kotsyurbenko O R, Friedrich M W, Simankova M V, et al. Shift from acetoclastic to $\mathrm{H}_{2}$-dependent methanogenesis in a West Siberian peat bog at low $\mathrm{pH}$ values and isolation of an acidophilic Methanobacterium strain. Appl Environ Microbiol, 2007, 4: 2344-2348

12 Kendall M M, Wardlaw G D, Bonin A S, et al. Diversity of Archaea in marine sediments from Skan Bay, Alaska, including cultivated methanogens, and description of Methanogenium boonei sp. nov. Int J Sys Evol Microbiol, 2007, 73: 407-414 
13 Kotsyurbenko O R, Chin K J, Glagolev M V, et al. Acetoclastic and hydrogenotrophic methane production and methanogenic populations in an acidic West-Siberian peat bog. Environ Microbiol 2004, 6: 1159-1173

14 Galand P E, Fritze H, Conrad R, et al. Pathways for methanogenesis and diversity of methanogenic archaea in three boreal peatland ecosystems. Appl Environ Microbiol, 2005, 71: 2195-2198

15 Zhang G, Tian J, Jiang N, et al. Methanogen community in Zoige wetland of Tibetan plateau and phenotypic characterization of a dominant uncultured methanogen cluster ZC-I. Environ Microbiol, 2008, 10: 1850-1860

16 Jiang N, Wang Y, Dong X. Methanol as the primary methanogenic and acetogenic precursor in the cold Zoige wetland at Tibetan Plateau. Microbial Ecol, 2010, 60: 206-213

17 Nichols D S, Miller M R, Davies N W, et al. Cold adaptation in the Antarctic archaeon, Methanococcoides burtonii, involves membrane lipid unsaturation. J Bacteriol, 2004, 186: 8508-8515

18 Roberts M F. Organic compatible solutes of halotolerant and halophilic microorganisms. Saline Sys, 2005, 1: 5

19 Lamosa P, Turner D L, Ventura R, et al. Protein stabilization by compatible solutes: Effect of diglycerol phosphate on the dynamics of Desulfovibrio gigas rubredoxin studied by NMR. Eur J Biochem, 2003, 270: 4606-4614

20 Matthias K. Compatible solute influence on nucleic acids: Many questions but few answers. Saline Sys, 2008, 4: 6

21 Yamanaka K. Cold shock response in Escherichia coli. J Mol Microbiol Biotech, 1999, 1: 193-202

22 Amato P, Christner B C. Energy metabolism response to low-temperature and frozen conditions in Psychrobacter cryohalolentis, Appl Environ Microbiol, 2009, 75: 711-718

23 Williams T J, Burg D W, Raftery M J, et al. Global proteomic analysis of the insoluble, soluble, and supernatant fractions of the psychrophilic archaeon Methanococcoides burtonii Part I: The effect of growth temperature. J Proteome Res, 2009, 9: 640-652

24 Thomas T, Kumar N, Cavicchioli R. Effect of temperature on the stability and activity of elongation factor 2 proteins from Antarctic and thermophilic methanogens. J Bacteriol, 2000, 182: 1328-1332

25 Saunders N F, Thomas T, Curmi P M, et al. Mechanisms of thermal adaptation revealed from the genomes of the Antarctic archaea Methanogeniu frigidum and Methanococcoides burtonii. Genome Res, 2003, 13: 1580-1588

26 Noon K R, Guymon R, Crain P F, et al. Influence of temperature on tRNA modification in archaea: Methanococcoides burtonii (optimum growth temperature $\left(T_{\mathrm{opt}}, 23^{\circ} \mathrm{C}\right)$ and Stetteria hydrogenophila $\left(T_{\mathrm{opt}}\right.$, $\left.95^{\circ} \mathrm{C}\right)$. Genome Res, 2003, 18: 5483-5490

27 Allen M A, Laura F M, Williams T J, et al. The genome sequence of the psychrophilic archaeon, Methanococcoides burtonii: the role of genome evolution in cold- adaptation. ISME J, 2009, 3: 1012-1035
28 Goodchild A, Saunders N F W, Ertan H, et al. A proteomic determination of cold adaptation in the Antarctic archaeon, Methanococcoides burtonii. Mol Microbiol, 2004, 53: 309-321

29 Goodchild A, Raftery M, Saunders N F W, et al. Cold adaptation of the Antarctic archaeon, Methanococcoides burtonii assessed by proteomics using ICAT, J Proteome Res, 2005, 4: 473-480

30 Burg D W, Lauro F M, Williams T J, et al. Analyzing the hydrophobic proteome of the Antarctic archaeon. Methanococcoides burtonii using differential solubility fractionation. J Proteome Res, 2010, 9: 664-676

31 Jenney F E, Verhagen M F J M, Cui X, et al. Anaerobic microbes: oxygen detoxification without superoxide dismutase. Science, 1999, 286: $306-309$

32 Giaquinto L, Curmi P M G, Siddiqui K S, et al. Structure and function of cold shock proteins in Archaea. J Bacteriol, 2007, 189: 5738-5748

33 Cavicchioli R, Thomas T, Curmi P M G. Cold stress response in archaea. Extremophiles, 2000, 4: 321-331

34 Nygard O, Nilsson L. Characterization of the ribosomal properties required for formation of a GTPase active complex with the eukaryotic elongation factor 2. FEBS, 2004, 179: 603-608

35 Thomas T, Cavicchioli R. Effect of temperature on stability and activity of elongation factor 2 proteins from Antarctic and thermophilic methanogens. J Bacteriol, 2000, 182: 1328-1332

36 Thomas T. Archaeal cold adapted proteins structural and evolutionary analysis of the elongation factor 2 proteins from psychrophilic, mesophilic and thermophilic methanogens. FEBS Lett, 1998, 439: 281286

37 Thomas T, Kmuar N, Cavicchioli R. Effects of ribosomes and intracellular solutes on activities and stabilities of elongation factor 2 proteins from psychrotolenrant and thermophilic methanogens. J Bacteriol, 2001, 183: 1974-1982

38 Lim J, Thomas T, Cavicchioli R. Low temperature regulated DEADbox RNA helicase from the Antarctic archaeon, Methanococcoides burtonii. J Mol Biol, 2000, 297: 553-567

39 Georgellis D, Sohlberg B, Hartl F U, et al. Identification of GroEL as a constituent of an mRNA-protection complex in Escherichia coli. Mol Microbiol, 1995, 6: 1259-1268

40 Ruggero D, Ciammaruconi A, Paola L. The chaperonin of the archaeon Sulfolobus solfataricus is an RNA-binding protein that participates in ribosomal RNA processing. EMBO J, 1998, 17: 3471-3477

41 Anantharaman V, Koonin E V, Aravind L, et al. TRAM, a predicted RNA-binding domain, common to rTNA uracil methylation and adenine thiolation enzymes. FEMS microbial Lett, 2001, 197: 215-221

42 Williams T J, Lauro F M, Ertan H, et al. Defining the response of a microorganism to temperatures that span its complete growth temperature range $\left(-2\right.$ to $\left.28^{\circ} \mathrm{C}\right)$ using multiplex quantitative proteomics. Environ Microbiol, 2011, 13: 2186-2203

Open Access This article is distributed under the terms of the Creative Commons Attribution License which permits any use, distribution, and reproduction in any medium, provided the original author(s) and source are credited. 